\title{
Painless hematuria and radio-opaque densities in the left renal area
}

\author{
Pankaj Dangle, MCh(Urology), MRCS;* Kanwar Gill, MRCP, FRCP; + \\ Chandra Shekhar Biyani, MS, D Urol, FRCS(Urol), FEBU*
}

\section{Abstract}

A 50-year-old woman presented with microscopic hematuria. Initial radiological investigations suggested the diagnosis of renal calculi in the left kidney. However, further assessment confirmed a renal artery aneurysm. We discuss the differential diagnosis of radio-opaque densities in the renal area.

Can Urol Assoc J 2009;3(3):E17-E20

\section{Résumé}

Une femme de 50 ans présente une hématurie microscopique. Les examens radiologiques initiaux portent à croire à la présence de calculs au rein gauche, mais les examens subséquents révèlent un anévrisme de l'artère rénale. Nous discutons ici du diagnostic différentiel en présence de zones de haute densité opaques aux rayons $X$ dans la région rénale.

\section{Introduction}

Renal artery aneurysm is a rare pathological entity and may manifest clinically with hypertension, blood in urine or abdominal pain. ${ }^{1}$ Calculous disease of the upper urinary tract constitutes one of the major indications for radiological evaluation of the genitourinary system. Although plain radiography may identify incidental calcified renal artery aneurysms, most are probably missed, as they are noncalcified. ${ }^{2}$ Therefore, digital subtraction angiography remains the gold standard for diagnosis.

\section{Case report}

A 50-year-old woman, pregnant with her second child, presented with an episode of self-limiting painless microscopic hematuria. She had no other urinary symptoms. Her medical and obstetrical history was unremarkable and she had no history of surgery. She was not taking any regular medication and smoked 20 cigarettes per day and drank 28 units of alcohol per week ( 1 UK unit is $10 \mathrm{~mL}$ or $8 \mathrm{~g}$ of pure alcohol). Her blood pressure was 118/81 mm Hg.
Clinical examination of the genitourinary system was normal and did not reveal any palpable mass or sounds. Results of routine blood investigations including serum creatinine (110 $\mu \mathrm{mol} / \mathrm{L})$ and urine cytology were normal. Flexible cystoscopy showed congested urothelium of the bladder, which contained debris. Ultrasonography of the renal tract revealed 2 calcifications in the left kidney with acoustic shadowing suggestive of renal calculi. The larger calcification was in the region of the renal pelvis and the smaller one was in a lower calyceal position (Fig. 1). Intravenous urography (IVU) control film showed 2 radio-opaque densities in the region of the left kidney (Fig. 2). Following intravenous contrast administration there was prompt uptake and excretion. A filling defect was noted in the left renal pelvis on the 15-minute postinjection film (Fig. 3).

As the density in the renal pelvis was much less radioopaque than the much denser calculus in the lower calyx and appeared attached to the medial wall of the pelvis on IVU, a ureteroscopy was performed to rule out a calcified tumour. On retrograde study the ureter appeared normal with a fixed renal pelvic filling defect, similar to that shown

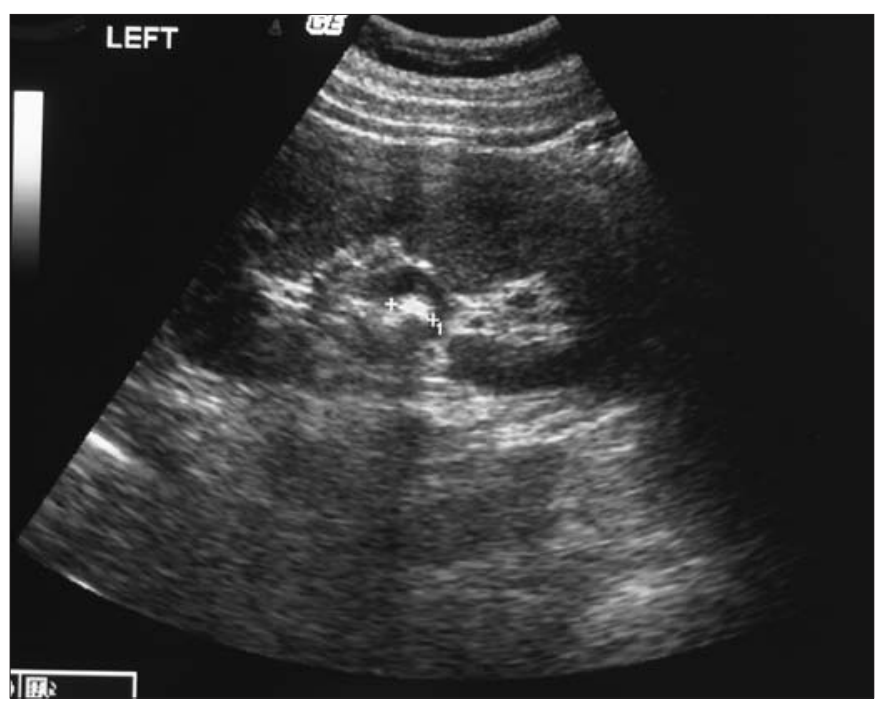

Fig. 1. Ultrasound of the left kidney demonstrating echogenic focus with distal acoustic shadowing in the region of the renal pelvis consistent with calcification. 
on IVU. Ureteroscopy showed a normal ureter and no lesion in the renal pelvis. A small bulge noted on the medial wall of the renal pelvis looked extrapelvic, with overlying normal mucosa (Fig. 4). Biopsy from the site showed normal urothelium. The patient was treated with extracorporeal shock wave lithotripsy for the lower calyceal stone. Repeat ultrasonography was organized to assess the densities in the kidney, which revealed no significant change in calcification. Repeat urine cytology was normal.

Because of the extrapelvic nature of the calcified lesion, a decision was made to perform computed tomography (CT) of the abdomen, which confirmed the 4-mm calculus in the lower pole calyx of the left kidney. The larger calcification was curvilinear and measured $10 \mathrm{~mm}$ with adjacent high-attenuation soft tissue suggesting calcification related to the left renal artery suspicious for renal artery aneurysm (Fig. 5).

Renal angiogram revealed normal right renal artery with

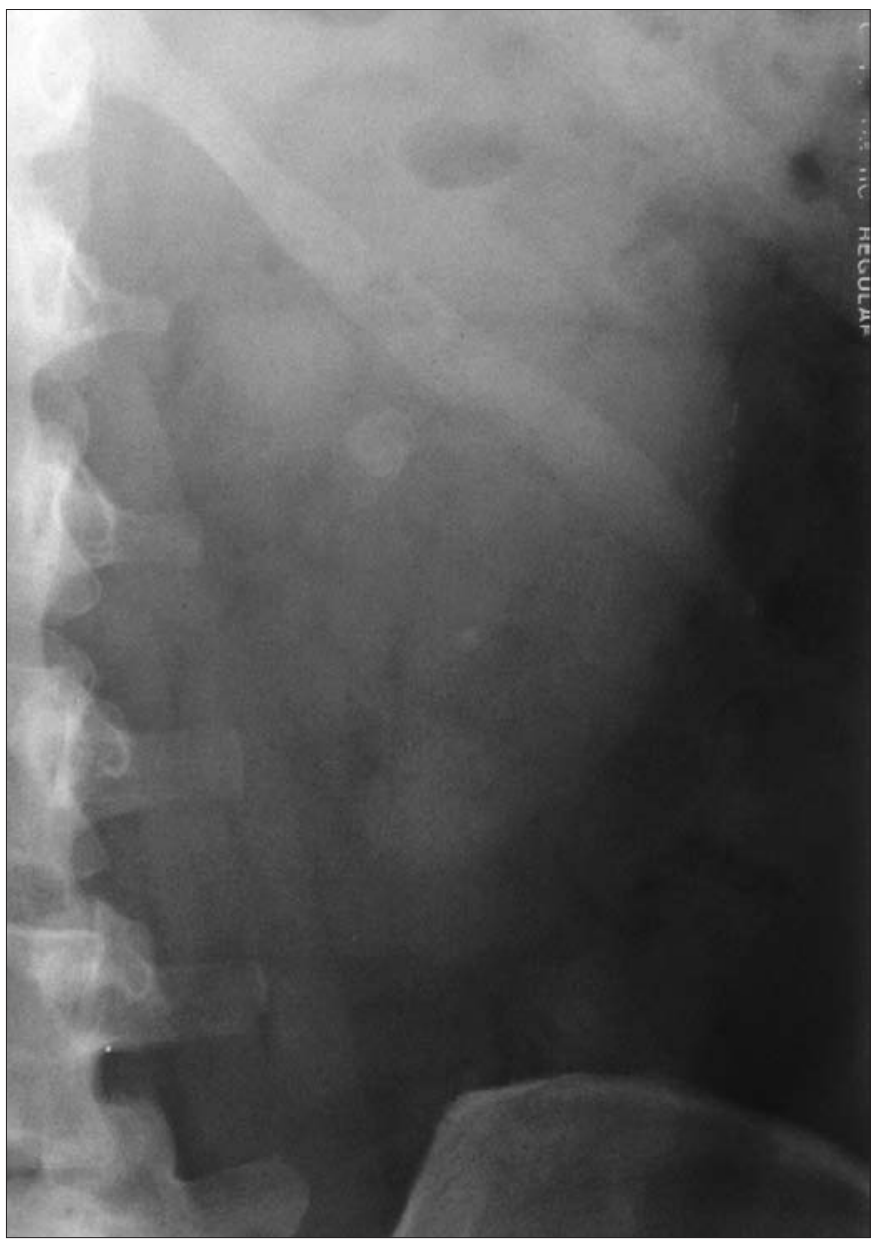

Fig. 2. Control film from intravenous urography series demonstrating 2 calcified opacities projected over the left renal outline. no aneurysm or stenosis. The left renal artery showed a saccular aneurysm arising from first-order lobar division. The distal artery was of normal calibre with no stenosis (Fig. 6). Vascular surgical opinion was sought and a conservative approach was advised.

\section{Discussion}

It is fairly common to see radio-opaque densities in the renal area on a plain abdominal film. The differential diagnosis of a radio-opaque density in the region of the left kidney on a plain abdominal radiograph includes the following:

- renal calculus

- calcified mesenteric lymph node

- tablets or foreign bodies in alimentary tract

- calcified blood vessel

- ossified tip of the 12th rib

- calcified tuberculous lesion in the kidney

- calcification within the left adrenal gland

- renal artery aneurysm

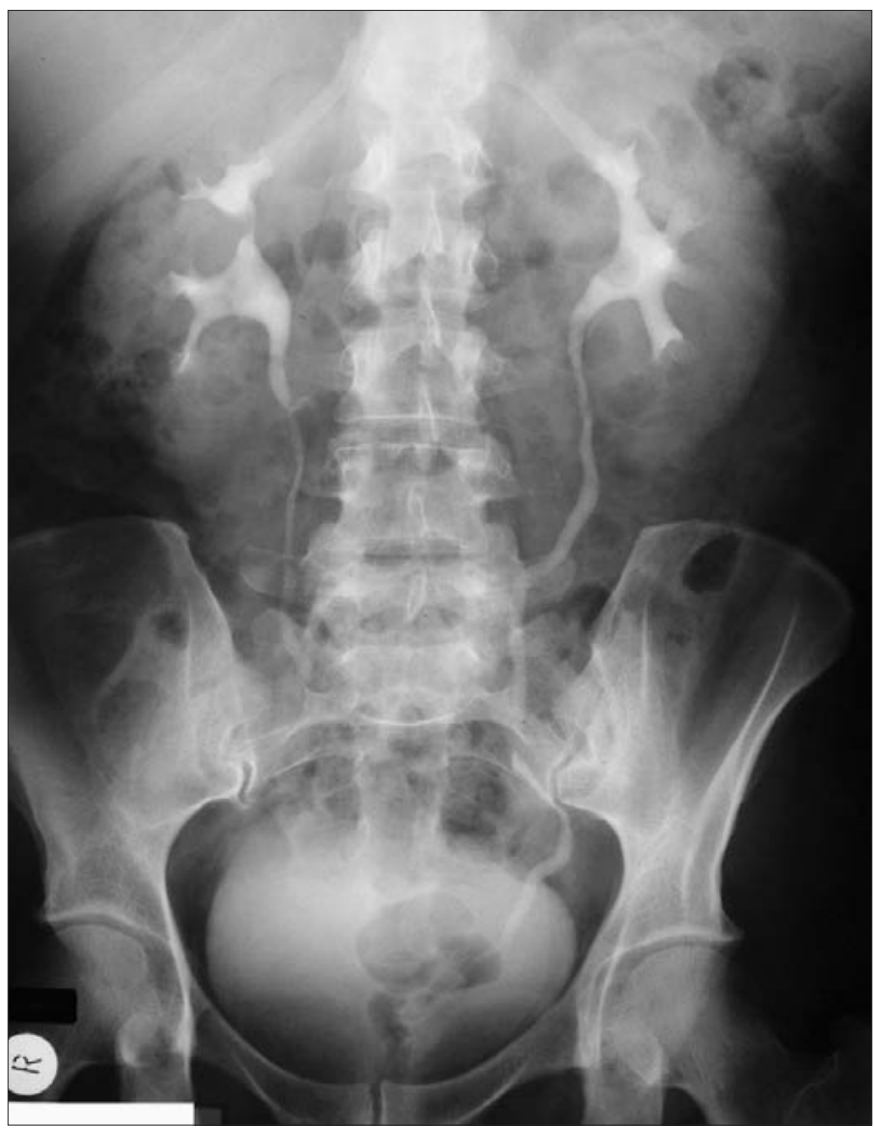

Fig. 3. Fifteen-minute film from the intravenous urography series revealing prompt excretion of contrast by both kidneys and a filling defect in the region of the left renal pelvis. 
It is of prime importance to assess the plain radiograph thoroughly before requesting any further investigation, as misinterpretation can lead to misdiagnosis. The density and type of calcification on a plain abdominal film should be assessed, especially in regard to whether it is within the pelvicalyceal system, parenchyma or outside the renal system. It is important to have a structured approach to the assessment of plain abdominal film to

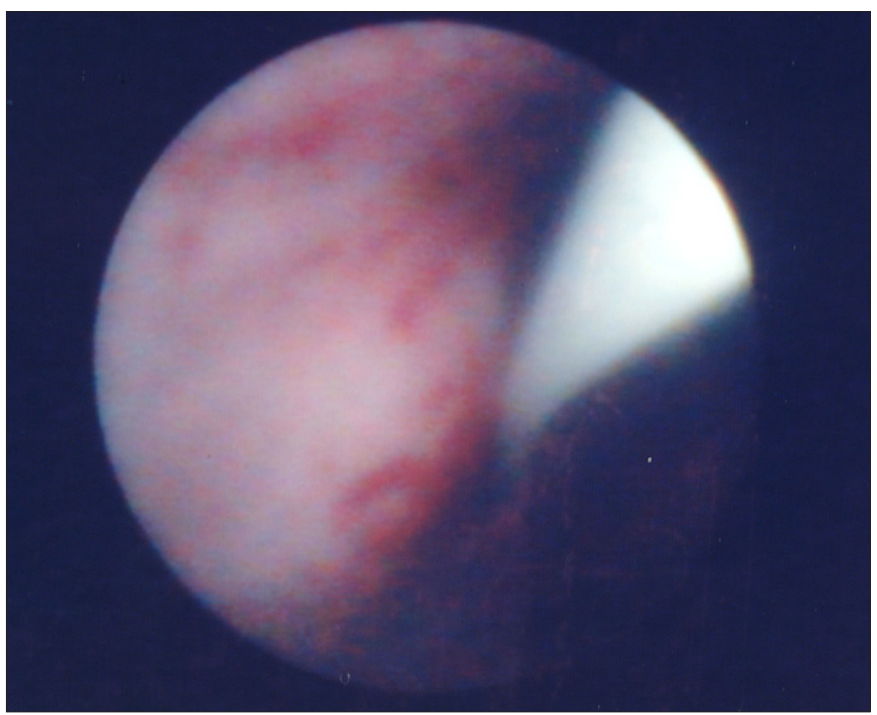

Fig. 4. Ureteroscopic view of the left renal pelvis showing a slight bulge on the medial wall of the renal pelvis suggestive of extrinsic compression.

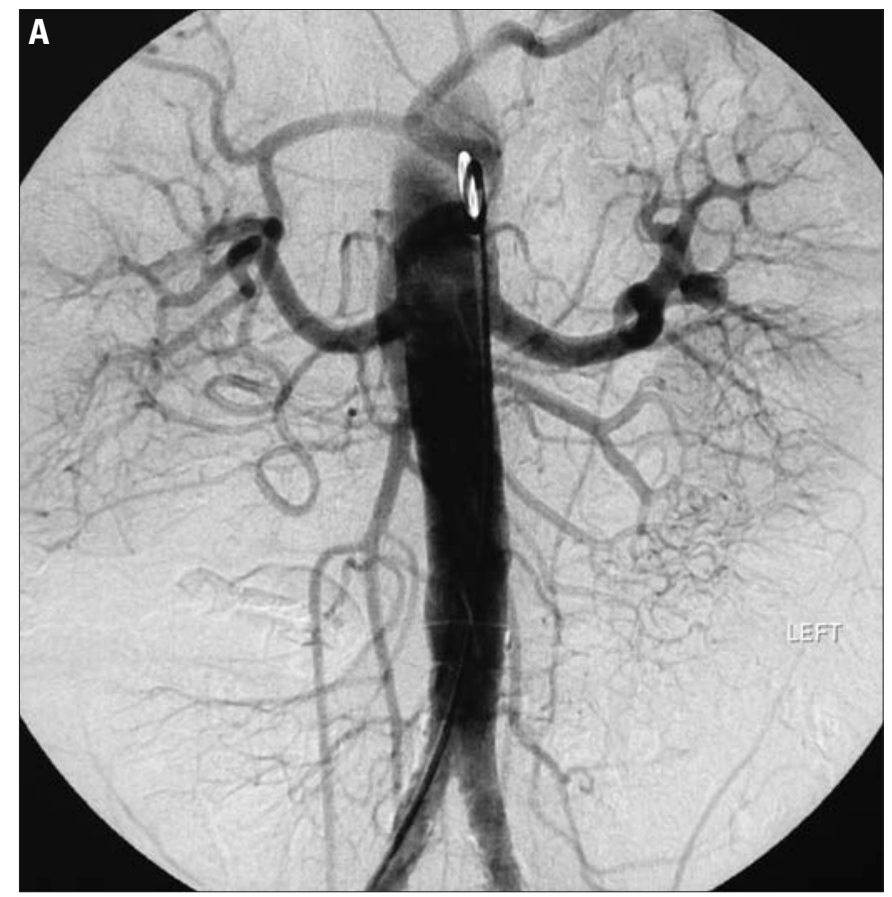

avoid misinterpretation. This can be accomplished by a) obtaining a radiograph in oblique position with the patient turned $30^{\circ}$ to $45^{\circ}$ to the side of the suspected density, b) the respiratory variation, c) plain film tomography and d) characteristic radiographic appearances (e.g., laminated features in the gallstone, curvilinear calcification with arterial calcifications). For any density that is different from its accompanying density, other potential

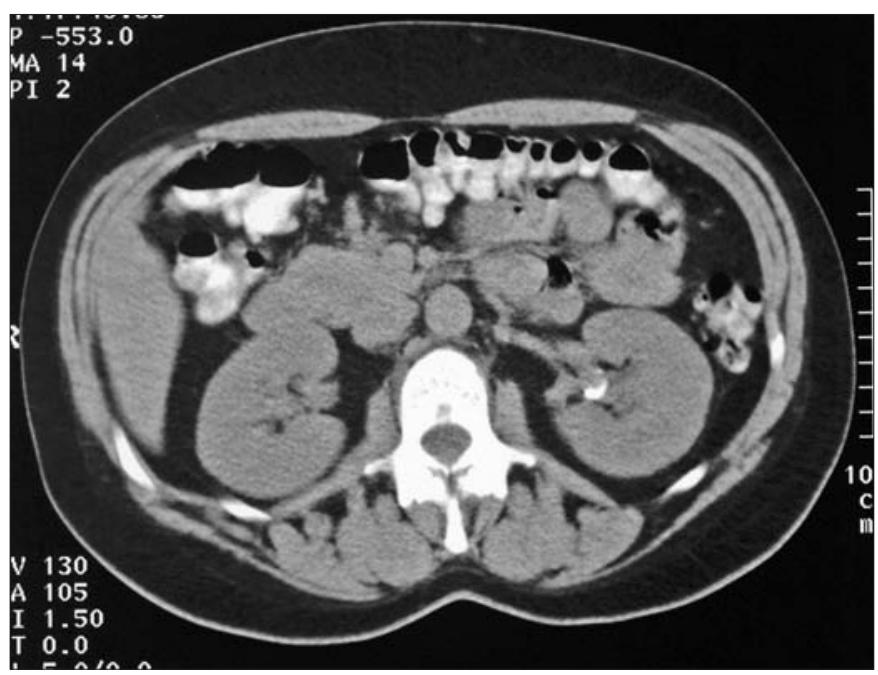

Fig. 5. Unenhanced computed tomography scan at the midrenal level demonstrating curvilinear calcification suggestive of a calcified left renal artery aneurysm.

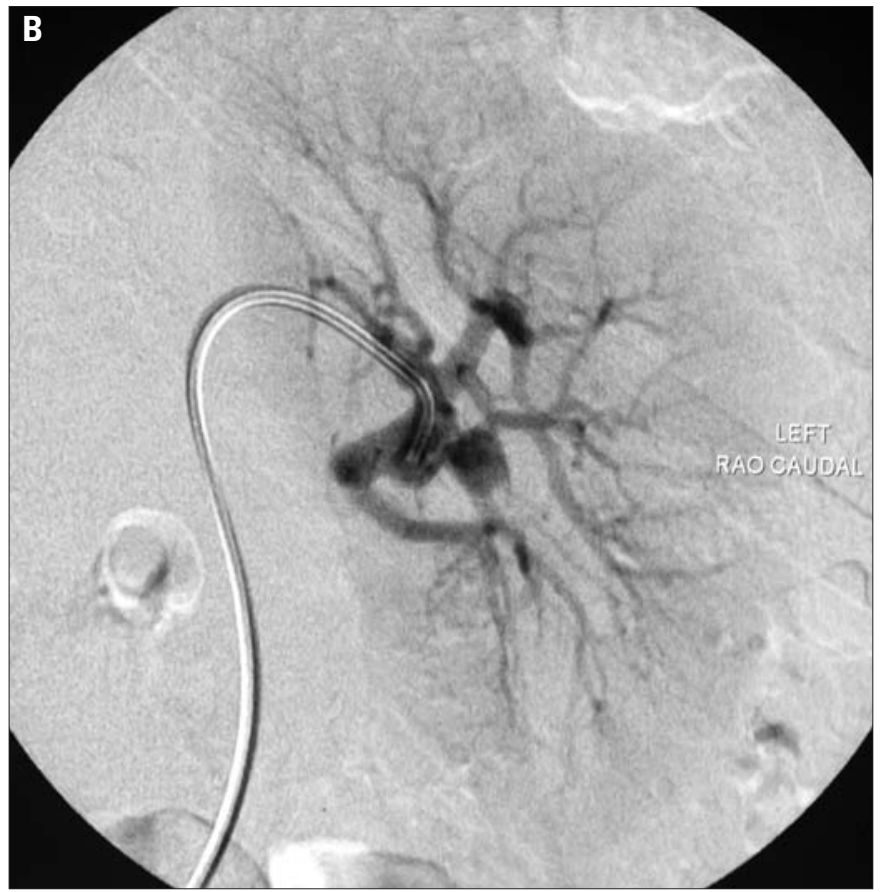

Fig. 6. Nonselective (A) and selective (B) renal angiography confirmed aneurysm arising from first lobar division of the left renal artery. 
diagnosis should be considered and appropriately investigated. However, with more widespread use of noncontrast CT to evaluate flank pain or radio-opacities over the renal area, these films may not be necessary. In our case initial imaging was done by IVU; this is a standard practice in most UK hospitals.

Renal artery aneurysms are relatively uncommon, being found in $0.09 \%$ of the general population. They rarely give rise to symptoms. As in this case they are usually incidental findings during investigation of patients with microscopic hematuria or high blood pressure.

Symptomatic renal artery aneurysms present most often with loin pain or, rarely, frank hematuria. Most patients are in the fourth or fifth decade of life and it is more common in women, with the right side more commonly affected. ${ }^{3,4}$

The fate of the aneurysm depends on the type. There are 4 types - saccular, fusiform, aneurysmal dissection and intrarenal microaneurysm. Of these the saccular variety is the most common. The exact cause is unknown but there is thought to be a congenital predisposition as cases have been reported in childhood. They are generally asymptomatic but may present with either the triad of pain, flank mass and hematuria, or any one of these symptoms. Gross hematuria is due to erosion of the aneurysm into the pelvicalyceal system whereas microscopic hematuria is unrelated to the aneurysm. ${ }^{5}$

Calcification of the aneurysm has been an unresolved issue to date as it is not clear whether calcification prevents rupture. In one of the largest series ${ }^{3}$ it has been observed that saccular shape rather than calcification is the reason for low prevalence for rupture.
There is a high incidence of rupture during pregnancy. The correlation between hypertension and aneurysm has still not been resolved. Most likely hypertension in these cases may be from renal ischemia secondary to thromboembolization distal to the aneurysm. ${ }^{6}$ The correlation may be a coincidental finding rather than the aneurysm being the cause of hypertension by microemboli to renal parenchyma as previously postulated. We suggest that in any unusual renal area calcification that does not look typical for renal calculus, aneurysm of the renal artery should be suspected.

From the Departments of *Urology and †Radiology, Pinderfields General Hospital, Wakefield, West Yorkshire, UK

This article has been peer reviewed.

Competing interests: None declared.

\section{References}

1. Hupp T, Allenburg JR, Post K, et al. Renal artery aneurysm: surgical indications and results. Eur J Vasc Surg 1992;6:477-86.

2. Mercier $C$, Piquet $P$, Piligian $F$, et al. Aneurysms of the renal artery and its branches. Ann Vasc Surg 1986; 1:321-7.

3. Henke PK, Cardneau JD, Welling TH III, et al. Renal artery aneurysms: a 35 year clinical experience with 252 aneurysm in 158 patients. Ann Surg 2001;234:454-63.

4. Serracino-Inglott F, Barnes N, Madan M. Renal artery aneurysm presenting with microscopic hematuria. J Vasc Surg 2003;38:620.

5. Hubert JP, Pairolcro PC, Kazmier FJ. Solitary renal aneurysm. Surgery 1980;88:557-65.

6. Youkey JR, Collins GJ Jr, Orecchia PM, et al. Saccular renal artery aneurysm as a cause of hypertension. Surgery 1985;97:498-501.

Correspondence: Mr. Chandra Shekhar Biyani, Department of Urology, Pinderfields General Hospital, Mid Yorkshire Hospitals NHS Trust, Aberford Rd., Wakefield, West Yorkshire UK WFI 4DG; fax +44 (0)1924 212921; shekharbiyani@hotmail.com 